\title{
Sonoanatomy of the spine: a comprehensive scanning protocol from cervical to sacral region
}

\author{
Ke-Vin Chang', Murat Kara², Daniel Chiung-Jui Su³ ${ }^{3}$ Eda Gürçay ${ }^{4}, B^{2}$ ram Kaymak², Wei- \\ Ting Wu${ }^{1}$, Levent Özçakar ${ }^{2}$
}

${ }^{1}$ Department of Physical Medicine and Rehabilitation National Taiwan University Hospital, Bei-Hu Branch and National Taiwan University College of Medicine, Taipei, Taiwan, ${ }^{2}$ Department of Physical and Rehabilitation Medicine, Hacettepe University Medical School, Ankara, Turkey, ${ }^{3}$ Department of Physical Medicine and Rehabilitation, Chi-Mei Medical Center, Tainan, Taiwan, ${ }^{4}$ Gaziler Training and Research Hospital, Department of Physical and Rehabilitation Medicine, Ankara, Turkey

\begin{abstract}
Ultrasound has been increasingly used in the musculoskeletal system, including the spine. In this protocol, the probe positionings, anatomical relationships, and ultrasound images of commonly scanned spinal structures are described. With an international consensus of several expert physiatrists i.e. USPRM; Ultrasound Study Group of ISPRM (International Society of Physical and Rehabilitation Medicine), this guide can provide a standardized approach for physicians who are interested in ultrasound diagnosis and guided pain interventions of the spine.

Keywords: vertebra; sonography; facet; pain; nerve root; intervention
\end{abstract}

\section{Introduction}

Ultrasound (US) imaging has been widely used to explore various musculoskeletal disorders for years. There is an increasing number of physicians who are capable of conducting ultrasonographic diagnoses and guided interventions on extremities $[1,2]$, and several protocols have been published [3-13]. Likewise, it is also paramount to have a systematic scanning protocol for the spine. This paper comprises regional anatomy and a description of the standardized scanning procedures on cervical, tho-

Received 20.05.2019 Accepted 26.07.2019

Med Ultrason

2019, Vol. 21, No 4, 474-482

Corresponding author: Levent Özçakar

Department of Physical and Rehabilitation

Medicine, Hacettepe University Medical

School, Ankara, Turkey

E-mail: lozcakar@yahoo.com racic and lumbosacral spine, and can serve as a practical guide for physicians who are interested in imaging and guided interventions of the spine (Table I).

\section{Anatomy}

\section{Bony Elements}

The human spine includes 7 cervical, 12 thoracic, 5 lumbar, and 5 sacral and 4 coccygeal coalesced vertebrae. A vertebra is composed of a vertebral body, two pedicles, two laminae, two transverse processes (TPs) and one spinous process (SP). The spinal canal, where the spinal cord is situated, is encircled by the vertebral bodies and the intervertebral discs anteriorly, the pedicles anterolaterally and the laminae posterolaterally. There are two openings of the spinal canal; the neural foramina bilaterally and interlaminar space posteriorly [14-16].

The atlas ( $\mathrm{C} 1$ vertebra) does not have a vertebral body. Instead, it has two big lateral masses joined by the 
anterior and posterior arches. The anterior arch is articulated with the odontoid process (dens), a bony projection from the vertebral body of the axis (C2 vertebra) [15].

The TPs are located anteriorly to the facet column at the cervical and lumbar spines and posteriorly to the facet column at the thoracic spine. The orientations of the facet joint interfaces differ in various levels. The superior articular processes at the upper cervical spine (C1 and $\mathrm{C} 2$ vertebrae) face upward and gradually transit to a posterior vertical orientation at the middle and lower cervical and upper thoracic regions. Their orientation further changes to posteromedial in the middle and lower thoracic and lumbar regions. Furthermore, regarding the cervical spine, the TPs from $\mathrm{C} 3$ to $\mathrm{C} 6$ vertebra levels have an anterior and a posterior tubercle, whereas the TP at C7 vertebra has only a posterior tubercle [14, $15]$.

The $\mathrm{SP}$ of $\mathrm{C} 1$ vertebra is absent or rudimentary, whereas that of C2's appears bifid. The C7 vertebra has a long, non-bifid SP, which is usually used as a landmark for counting the vertebra levels. The SPs of cervical and upper thoracic vertebrae project directly posteriorly, whereas those of the middle thoracic vertebrae extend posteriorly with a significant downward angle. The SPs of the lowermost thoracic (T9-12) and lumbar spine stick out posteriorly with a slightly inferior angulation [14-16].

\section{Ligamentous structures}

The supraspinous ligament bridges the tips of adjacent SPs with a connection of the nuchal ligament. It is located superficial to the interspinous ligament. The ligamentum flavum spans over the interlaminar space. Between the ligamentum flavum and the posterior dura is the epidural space. Anterior to the posterior dura is the thecal space, the ventral border of which is the anterior dura and posterior longitudinal ligament. The anterior aspect of the vertebral bodies is reinforced by the anterior longitudinal ligament. The intertransverse ligaments are positioned between the neighboring TPs and are more prominent in the thoracic and lumbar spines. In the cervical region, the intertransverse ligaments are scarcely arranged fibers and are usually replaced by muscles [14-16].

In the thoracic spine, there is another group of ligaments worth noticing. The superior costal transverse ligament arises from the TPs and attaches to the neck of the rib in one intercostal space below. The lateral costal transverse ligament originates from the posterior surface of the TP and extends laterally to insert on the tubercle of the rib at the same level [14-16].

Table I. Summary of important anatomic structures, subject/transducer positions and relevant ultrasonographic findings of the spine

\begin{tabular}{|c|c|c|c|}
\hline Structure & Posture & Transducer & Ultrasonographic finding \\
\hline \multicolumn{4}{|l|}{ Cervical spine } \\
\hline Nerve roots & $\begin{array}{l}\text { Supine, head rotating } \\
\text { to the contralateral side }\end{array}$ & $\begin{array}{l}\text { Horizontal plane, } \\
\text { anterior lateral neck }\end{array}$ & $\begin{array}{l}\text { Hypoechoic bubbles emerging from the inter-tubercular } \\
\text { grooves of the transverse process }\end{array}$ \\
\hline Sympathetic ganglion & $\begin{array}{l}\text { Supine, head rotating } \\
\text { to the contralateral side }\end{array}$ & $\begin{array}{l}\text { Horizontal plane, } \\
\text { anterior lateral neck }\end{array}$ & $\begin{array}{l}\text { Hypoechoic structures located deep to the prevertebral } \\
\text { fascia, superficial to the longus coli muscle }\end{array}$ \\
\hline Medial branch & Side-lying & $\begin{array}{l}\text { Coronal plane, lateral } \\
\text { neck }\end{array}$ & $\begin{array}{l}\text { Hypoechoic bubbles located at the waists of the cervical } \\
\text { facet column }\end{array}$ \\
\hline Greater occipital nerve & Prone & $\begin{array}{l}\text { Horizontal plane, } \\
\text { posterior neck }\end{array}$ & $\begin{array}{l}\text { Hypoechoic bubbles interposed between semispinalis } \\
\text { capitis and obliquus capitis inferior muscles }\end{array}$ \\
\hline \multicolumn{4}{|l|}{ Thoracic spine } \\
\hline Intercostal space & Prone & $\begin{array}{l}\text { Sagittal plane, slightly } \\
\text { lateral to the midline, } \\
\text { upper back }\end{array}$ & $\begin{array}{l}\text { Space between two ribs and superficial to the parietal } \\
\text { pleura }\end{array}$ \\
\hline Paravertebral space & Prone & $\begin{array}{l}\text { Horizontal plane, } \\
\text { between two ribs near } \\
\text { the midline, upper back }\end{array}$ & $\begin{array}{l}\text { Wedge-shape space bordered by the vertebrae, } \\
\text { parietal pleura and intercostal membrane }\end{array}$ \\
\hline \multicolumn{4}{|l|}{ Lumbar spine } \\
\hline Facet joint & Prone & $\begin{array}{l}\text { Sagittal plane, slightly } \\
\text { lateral to the midline, } \\
\text { lower back }\end{array}$ & $\begin{array}{l}\text { Facet column appears as 'camel humps' with gaps } \\
\text { interposed between the superior and inferior articular } \\
\text { processes of adjacent levels }\end{array}$ \\
\hline Medial branch & Prone & $\begin{array}{l}\text { Horizontal plane, } \\
\text { slightly lateral to the } \\
\text { midline, lower back }\end{array}$ & $\begin{array}{l}\text { Structure located at the intersection of the superior } \\
\text { articular and transverse processes }\end{array}$ \\
\hline \multicolumn{4}{|l|}{ Sacral spine } \\
\hline Caudal epidural space & Prone & Sagittal plane, midline & $\begin{array}{l}\text { Hypoechoic space underneath the sacrococcygeal } \\
\text { ligament and above the sacrum }\end{array}$ \\
\hline
\end{tabular}




\section{Neural components}

The spinal cord is enveloped by meninges (pia, arachnoid and dura) and ends at the levels between L1 and L2 vertebrae in adults. The dural sac terminates at the S2 level. The spinal cord gives off spinal nerves which divide into two major branches; ventral and dorsal rami. The ventral rami innervate the extremities and anterolateral parts of the trunk. The dorsal rami further give off the medial, intermediate and lateral branches to innervate epaxial muscles such as multifidus, longissimus andiliocostalis. The medial branch also provides innervation to the facet joints.

Regarding the courses of the spinal nerves, the spinal nerves at or higher than the $\mathrm{C} 7$ level run above the TPs of the corresponding vertebrae after they exit the intervertebral foramen. On the other hand, the spinal nerves at or lower than the T1 level course below the TPs of the corresponding vertebra. Since there is no C8 vertebra, the C8 spinal nerve advances underneath the TP of C7 vertebra [15].

\section{Vascular network}

In the cervical region, the important vessels include common carotid artery, internal jugular vein, vertebral, inferior thyroid, transverse cervical and deep cervical arteries. The common carotid artery and internal jugular vein course inside the carotid sheath with the vagus nerve. The vertebral artery originates from the subclavian artery and enters the foramen transversalis of the TPs mostly at the $\mathrm{C} 6$ vertebral level. The inferior thyroid and transverse cervical arteries both arise from the thyrocervical trunk. The former runs into the thyroid gland through its lower pole, while the latter courses below the inferior belly of the omohyoid muscle and supplies trapezius, levator scapulae and rhomboid muscles. The deep cervical artery emerges from the costocervical trunk and courses between the semispinalis capitis and cervicis muscles near the cervical facet column [17,18].

In the thoracic and lumbar regions, the aorta gives off posterior intercostal and lumbar arteries. The spinal cord is supplied by the anterior and posterior radicular arteries, both of which are derived from the spinal branches of the posterior intercostal and lumbar arteries. The largest anterior radicular artery is named the "Adamkiewicz artery" [18].

\section{Scannıng technique}

\section{Cervical spine}

\section{Anterolateral aspect}

The transducer is placed at the supraclavicular fossa along the axial plane. The first rib, appearing as a hyperechoic straight line, is interposed by the hyperreflective movable pleura from the anterior and posterior aspects. Brachial plexus is located above the first rib, looking like a bunch of grapes. The transducer is relocated more cranially along the anterolateral aspect of the cervical spine. After passing the interscalene region, where the brachial plexus is sandwiched between the scalenus anterior and scalenus medius muscles (fig 1A), the $\mathrm{C} 7$ nerve root and $\mathrm{TP}$ of $\mathrm{C} 7$ vertebra (with a prominent posterior tubercle and a small and inconspicuous anterior tubercle) can be visualized (fig 1B). Anterior to the $\mathrm{C} 7$ nerve root is the vertebral artery, which can be enhanced by Doppler imaging. Moving the transducer more cranially, the TP of C6 vertebra (with a prominent anterior tubercle, also named Chassaignac tubercle, and a regular-sized posterior tubercle) can be visualized with the $\mathrm{C} 6$ nerve root emerging from the intertubercular groove (fig 1C). Relocating the transducer more cranially, the TP of $\mathrm{C} 5$ vertebra (with similar sized anterior and posterior tubercles) appears with $\mathrm{C} 5$ nerve root arising between the tubercles (fig 1D) $[19,20]$. Once the short-axis views of the nerve roots are located, the transducer can be pivoted to the coronal oblique plane to visualize the nerve roots in their long-axis (fig 2).

Relocating the transducer more ventrally along the axial plane, the vertebral body with a hyperechoic arched bony cortex can be visualized adjacent to the TP. The longus coli muscle is seen on the anterolateral surface of the vertebral column (fig 1). Moving the transducer more cranially to the $\mathrm{C} 6$ vertebral level, the longus capitis emerges superolateral of the longus coli muscle (fig $1 \mathrm{C}, \mathrm{D})$.

The prevertebral fascia is located posterior to the carotid sheath and in front of the prevertebral muscles. The space between the prevertebral fascia and muscles is where there is the sympathetic chain and is also the fascia plane for the stellate ganglion block (fig 3) [19,21].

\section{Lateral aspect}

The transducer is placed along the coronal plane on the mastoid process. The TP of $\mathrm{C} 1$ vertebra (at the same coronal plane of the atlanto-occipital and atlanto-axial joints) can be seen caudal to the mastoid process as a small hyperechoic bony curvature. Since the atlanto-occipital and atlanto-axial joints are located anterior to the facet column of the cervical spine, posterior relocation of the transducer facilitates visualization of the facet joint between the inferior articular process of the $\mathrm{C} 2$ vertebra and superior articular process of the $\mathrm{C} 3$ vertebra, where the third occipital nerve courses above (fig 4A). Moving the transducer caudally, the cervical facet column appears as a mountain range. The mountain peaks represent the facet joints, while the valleys are the locations for the medial branches (fig 4B). Since the TP of C7 vertebra is 

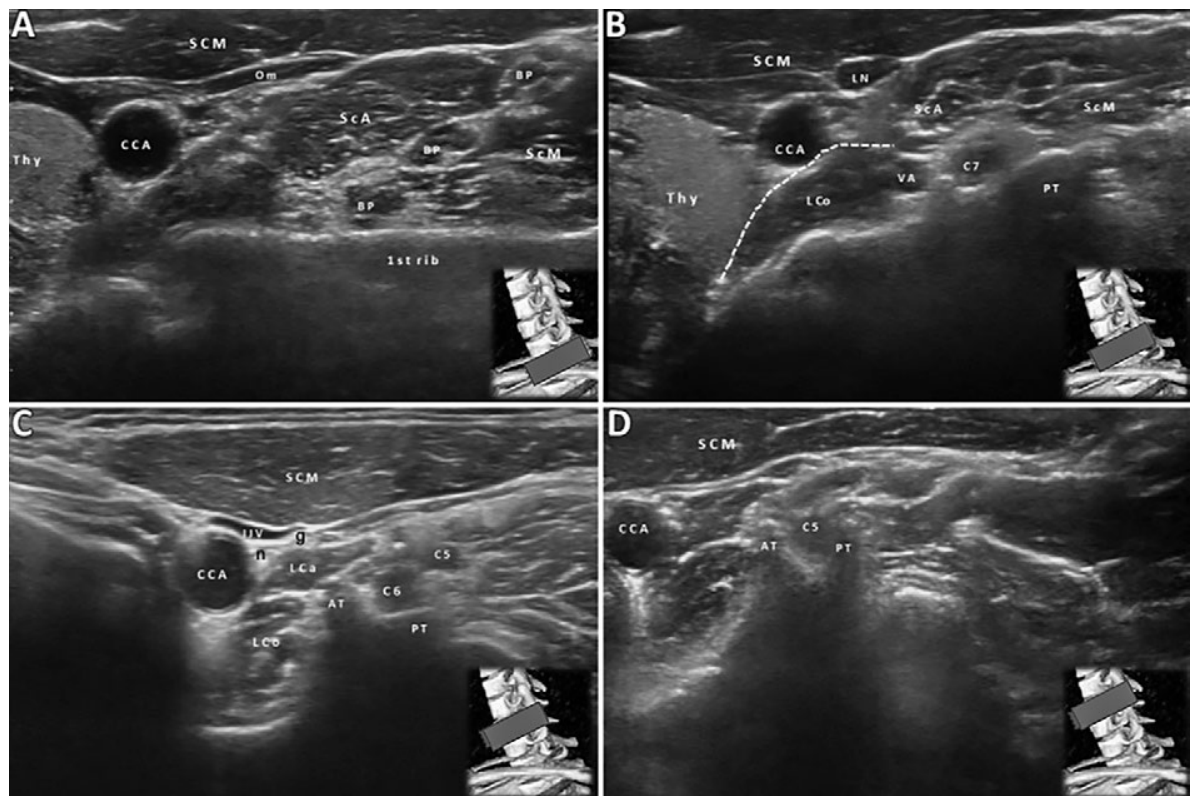

Fig 1. Axial ultrasound imaging of cervical nerve roots and the brachial plexus at the supraclavicular (A), C7(B), C6 (C), and C5 (D) vertebrae levels. SCM, sternocleidomastoid muscle; Thy, thyroid gland; Om, omohyoid muscle; CCA, common carotid artery; ScA, scalenus anterior muscle; BP, brachial plexus; ScM, scalenus medius muscle; LCo, longus colli muscle; LCa: longus capitis muscle;IJV, internal jugular vein; LN, lymph node; VA, vertebral artery; PT, posterior tubercle; AT, anterior tubercle; n, vagal nerve; g, cervical sympathetic ganglion; dashed line, prevertebral fascia
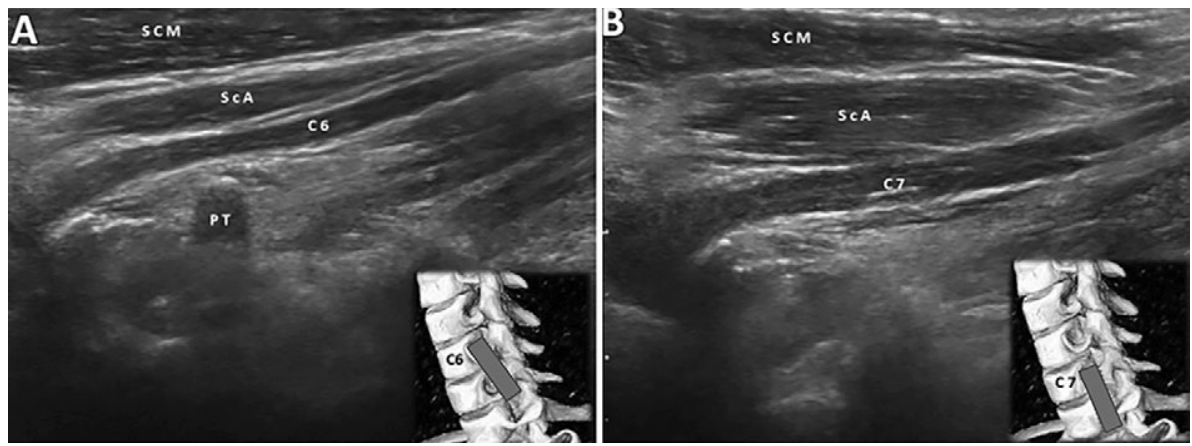

Fig 2. Coronal obliqueultrasound imaging of the C6 (A) and C7 (B) nerve roots in the long-axis views. SCM, sternocleidomastoid muscle; ScA, scalenus anterior muscle; PT, posterior tubercle

very close to the articular pillar, the $\mathrm{C} 7$ medial branch is situated at the base of TP of C7 vertebra (fig 4C), unlike the typical locations of other cervical medial branches (in the middle between the adjacent facet joints) [21,22].

\section{Posterior aspect}

The transducer is placed along the axial plane on the occiput and is then relocated caudally to visualize the posterior arch of atlas ( $\mathrm{C} 1$ vertebra), appearing as a hemicircular hyperechoic bony surface (fig 5A). Moving the transducer caudally and obliquely, the long-axis view of the greater occipital nerve can be seen within the fascia between the muscles semispinalis capitis muscle (above) and oblique capitis inferior (below) (fig 5B). The lateral edge of the transducer can be pivoted one vertebral level cranially to reach to the lateral mass of $\mathrm{C} 1$ vertebra. The quadrilateral-shaped muscle connecting the SP of $\mathrm{C} 2$ vertebra and lateral mass of $\mathrm{C} 1$ vertebra is the obliquus capitis inferior muscle (fig 5C). The greater occipital nerve can be visualized as a hypoechoic round structure in the short-axis view $[19,23]$.

\section{Thoracic spine}

Correct localization of the thoracic vertebral levels can be achieved by counting the corresponding ribs. Placing the transducer in the sagittal plane on the supraclavicular fossa at the junction between the neck and upper shoulder, the first rib is easily identified as a hyperechoic bony plane with acoustic shadowing. Once it is precisely 


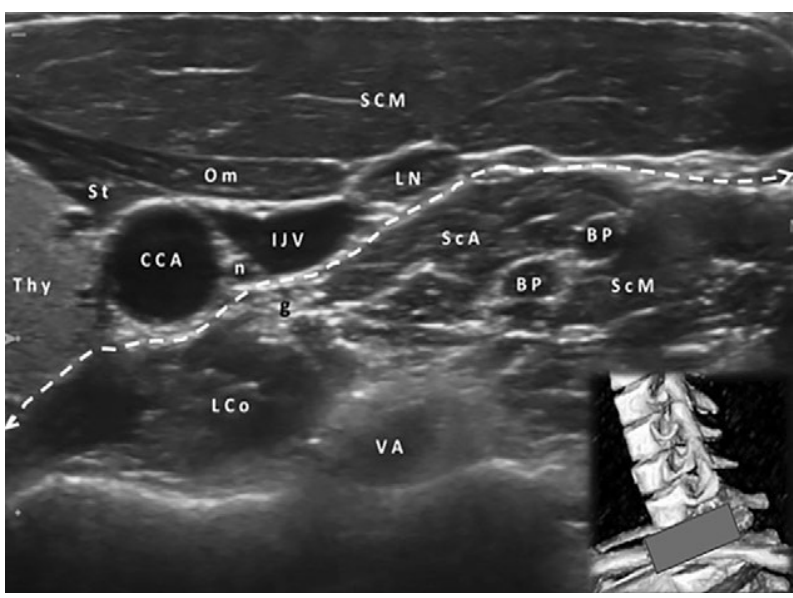

Fig 3. Axial ultrasound imaging of the stellate ganglion $(\mathrm{g})$ and the prevertebral fascia (dashed line). The patient's neck is rotated away from the transducer, which is placed horizontally at the C7 level. SCM, sternocleidomastoid muscle; Thy, thyroid gland; Om, omohyoid muscle; CCA, common carotid artery; $\mathrm{ScA}$, St; sternothyroid muscle; scalenus anterior muscle; BP, brachial plexus; ScM, scalenus medius muscle; LCo, longus colli muscle; IJV, internal jugular vein; LN, lymph node; VA, vertebral artery; n, vagal nerve located, other ribs can be recognized sequentially by relocating the transducer more caudally [24,25].

After the desired rib level is seen (fig 6A), moving the transducer more medially allows visualization of the TP (fig 6B), whereby its bony cortex is more superficial (posterior) to the ribs. Deep (anterior) to the TPs, the superior costal transverse ligament is seen bridging the TP and the neck of the rib (one vertebral level below). Relocating the transducer further medially, the facet joint is seen between the two hyperechoic lines (inferior articular process of the above vertebra and superior articular process of the below vertebra) (fig 6C). Since the laminae of the thoracic vertebrae are overlapping, it is not easy to differentiate the facet columns from the interlaminar spaces. Positioning the transducer more centrally, the tips of the SPs are seen linearly arranged and are linked by the supraspinous and interspinous ligaments (fig 6D) [24,25].

The transducer can be pivoted 90 degrees to also scan the thoracic spine in the axial plane. The lateral costal transverse ligament bridges the costotransverse joint to connect the rib and the corresponding TP (fig 7A). Me-
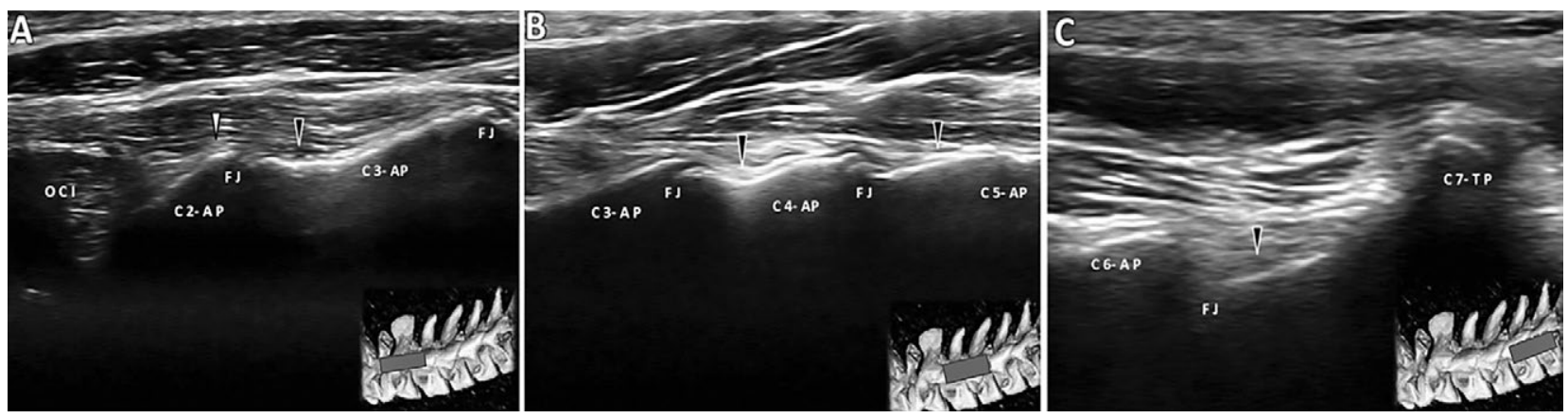

Fig 4. Coronal ultrasound imaging of upper (A), middle (B) and lower (C) cervical facet columns. OCI, obliquus capitis inferior muscle; AP, articular pillar; FJ, facet joint; white arrowhead, third occipital nerve; black arrowhead, cervical medial branch; TP, transverse process
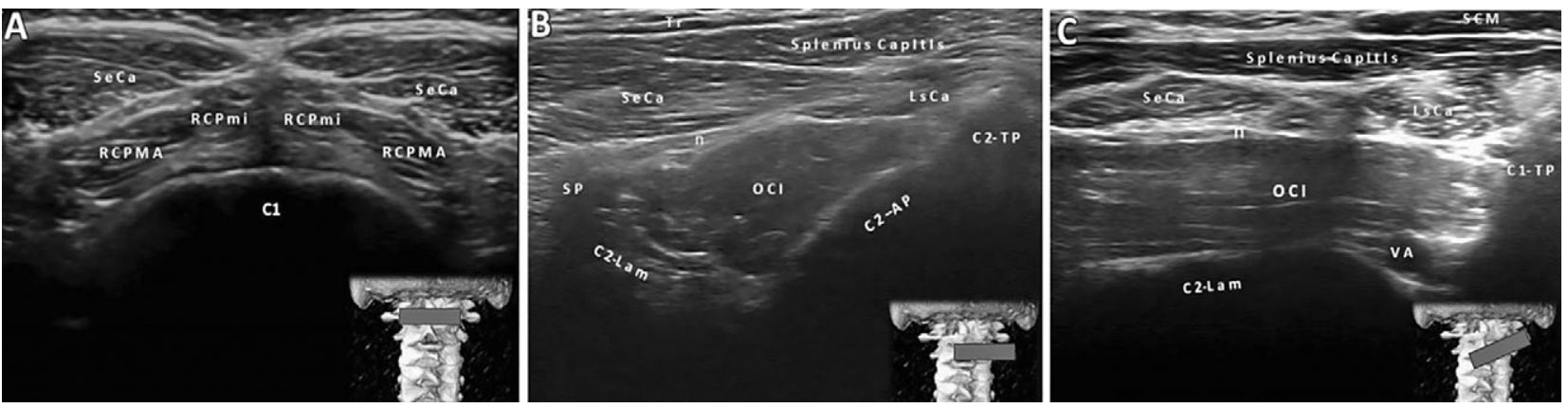

Fig 5. Axial ultrasound imaging at the occiput-C1 level (A), axial (oblique) view at the $\mathrm{C} 1-\mathrm{C} 2$ vertebral level (B) and along the longaxis of the obliquus capitis inferior (OCI) muscle (C). OCI; obliquus capitis inferior muscle; SeCa, semispinalis capitis; RCPMj, rectus capitis posterior major; RCPMn, rectus capitis posterior minor; Tr, trapezius; $\mathrm{n}$, greater occipital nerve; LsCa, longissimus capitis muscle; SP, spinous process; Lam, lamina; AP, articular pillar; TP, transverse process; VA, vertebral artery; SCM, sternocleidomastoid muscle 

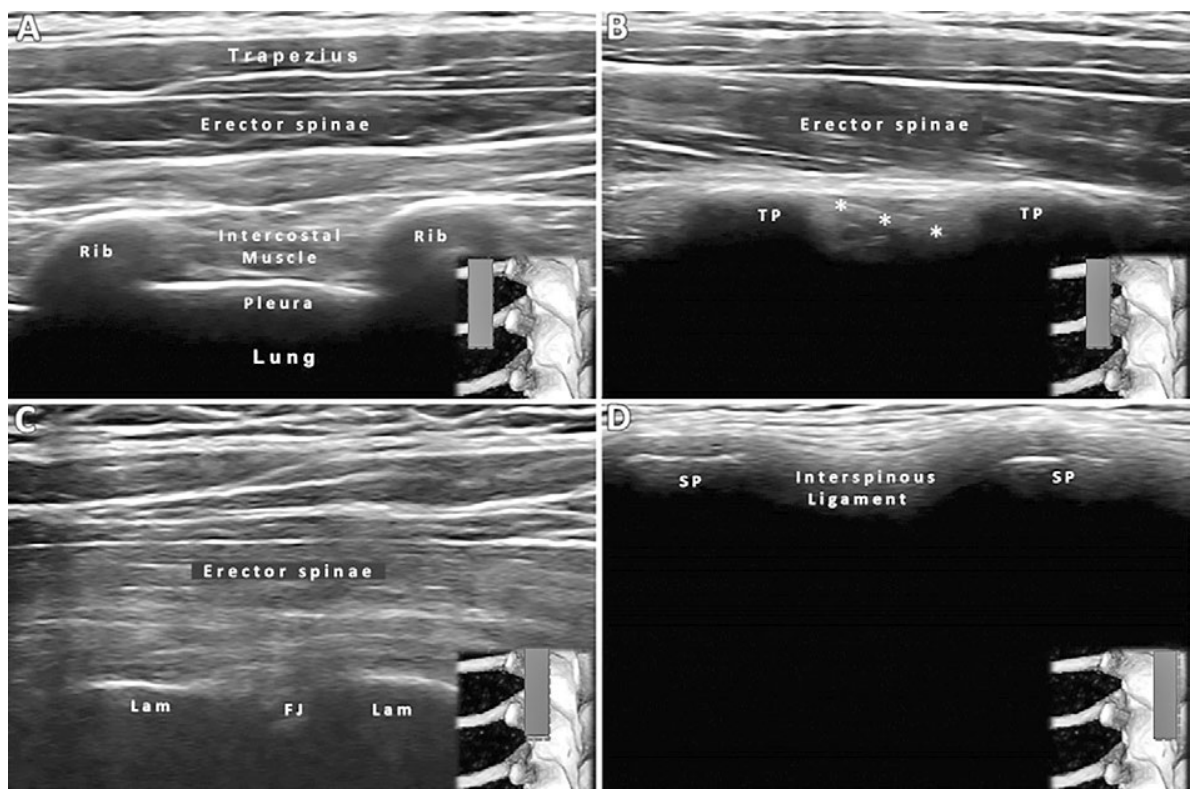

Fig 6. Sagittal ultrasound imaging of the thoracic spine at the levels of ribs (A), transverse processes (B), facet columns (C), and spinous process (D). TP, transverse process; *, superior costotransverse ligament; FJ, facet joint; Lam, lamina; SP, spinous process
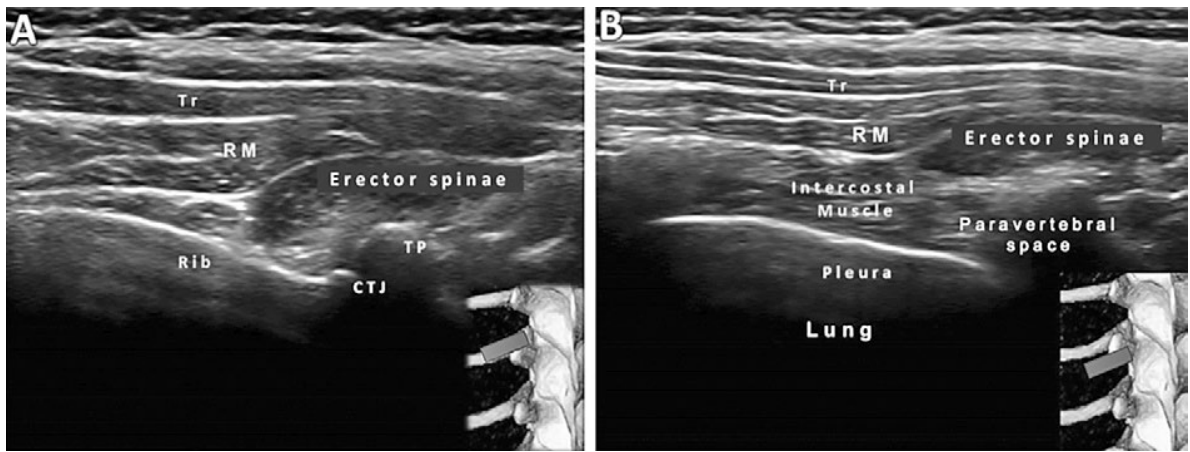

Fig 7. Axial ultrasound imaging of the thoracic spine at the levels of rib (A) and intercostal space (B). Tr, trapezius muscle; TP, transverse process; CTJ, costotransverse joint; RM, rhomboid major muscle

dial to the costotransverse joint is the curved bony plane conjoining the TP, lamina and SP, seeming like one side of the bird wings. Moving the transducer more cranially or caudally, the intercostal space is seen lateral to the TP or lamina (fig 7B). This area is also terminated as the thoracic paravertebral space, bordered by the pleura anteriorly and the superior costal transverse ligament posteriorly $[24,25]$.

\section{Lumbar Spine}

The transducer is placed along the sagittal plane in the midline of the lumbar region, enabling to visualize the SPs linked by the supraspinous ligaments superficially and interspinous ligaments deeply (fig 8A). Relocating the transducer laterally, the laminae (resembling 'horse heads') and the interlaminar spaces can be seen (fig 8B).
Pivoting the transducer toward the midline to let the US beam be more perpendicular to the laminae, the epidural space between the dura and ligamentum flavum can be clearly recognized [24,25].

Relocating the transducer more laterally, the facet column (appearing as 'camel humps') can be visualized (fig 8C). More lateral are the TPs, which cast acoustic shadowings on the psoas major muscle, also known as the "the trident sign" (fig 8D). The lumbar plexus, having some fibrillary hyperechoic structures, can be seen in the posterior aspect of the psoas major muscle. However, since the intramuscular tendons can mimic the lumbar plexus, electric stimulation can be used for confirmation [24,25].

Regarding the axial scanning of the lumbar spine, the transducer can first be placed on the cranial end of the sa- 

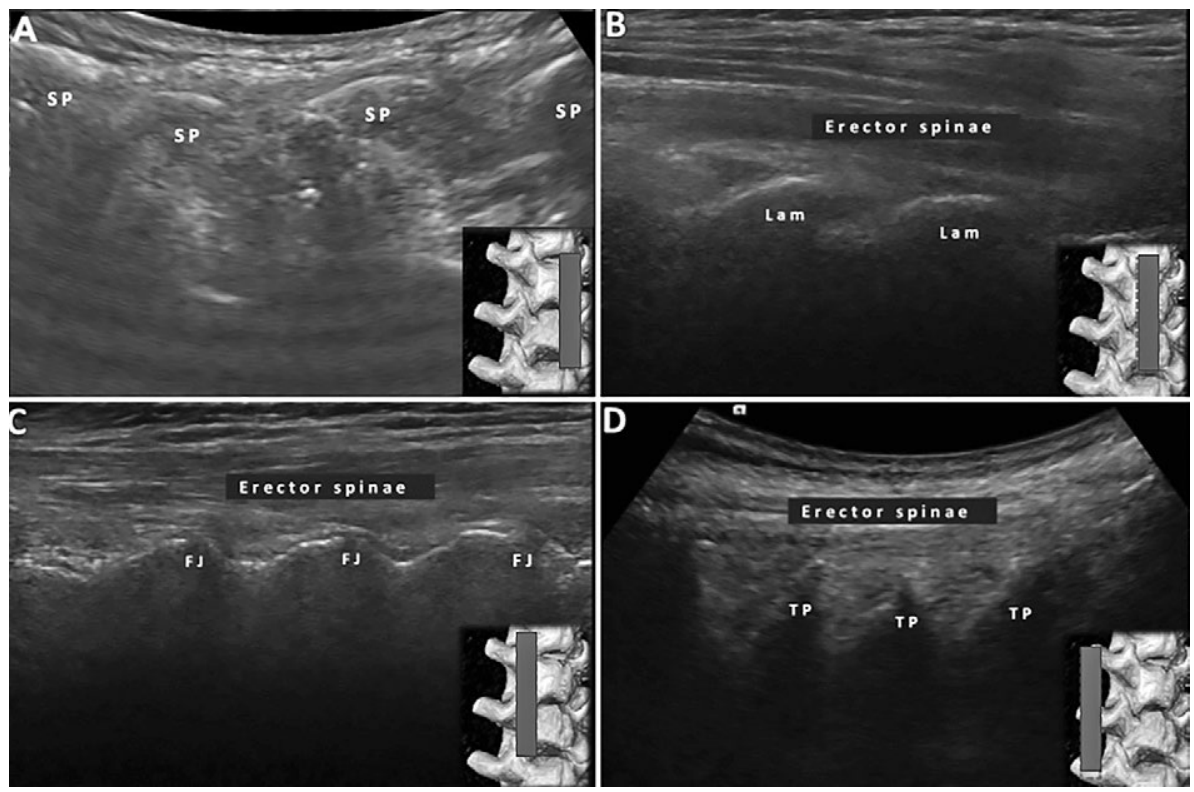

Fig 8. Sagittal ultrasound imaging of the lumbar spine at the spinous process (A), lamina (B), facet column (C) and transverse process (D) levels. SP, spinous process; Lam, lamina; FJ, facet joint; TP, transverse process
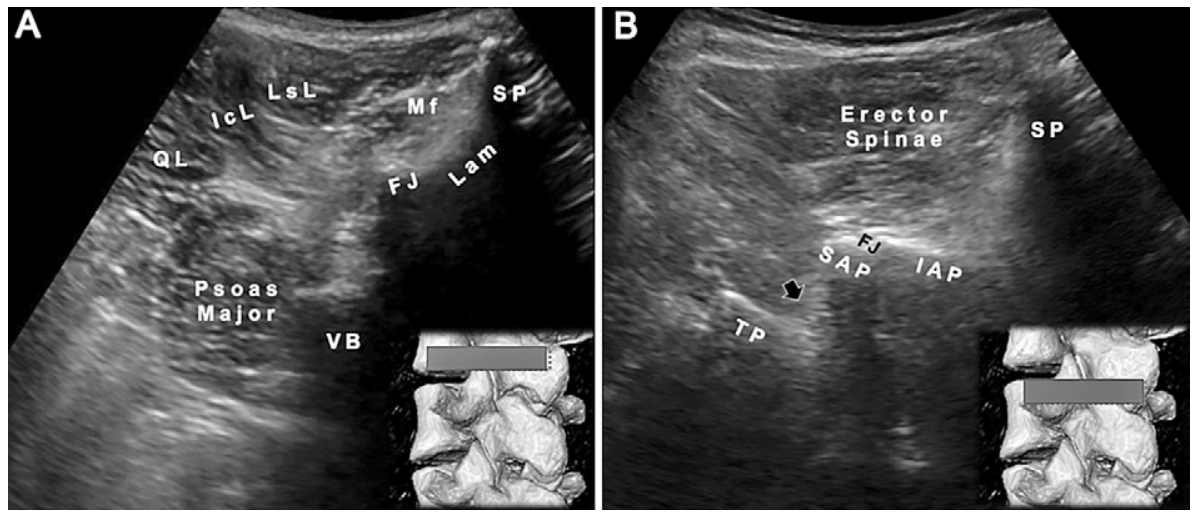

Fig 9. Axial ultrasound imaging of the lumbar spine at the intertransverse (A) and transverse (B) process levels where the arrow points towards the medial branch. SP, spinous process; Lam, lamina; TP, transverse process; VB, vertebral body; Mf; multifidus muscle; IcL; iliocostalis lumborum muscle; LsT; Longissimus lumborum muscle, QL, quadratus lumborum muscle; FJ, facet joint; SAP, superior articular process; IAP, inferior articular process

crum with two oblique bony planes joined in the midline. Moving the transducer more cranially, the L5/S1 interlaminar space is reached and the L5/S1 facet joints can be seen. The L5 medial branch is located at the junction between the S1 vertebra superior articular process and the sacral ala. Advancing the transducer further cranially, the L4/L5 interlaminar space is visualized. The L4 medial branch is coursing over the corner interposed by the bases of the L5 superior articular process and TP of the L 5 vertebra. The other lumbar facet joints and medial branches can be approached as mentioned above (fig 9) [24,25]. Additionally, throughout the scanning, epaxial muscles can be identified (individually) where necessary [26].

\section{Sacral Spine}

The transducer is placed along the sagittal plane in the midline on the L5/S1 inter-laminar space (fig 10A). The sacrum appears as an oblique bony plane which gradually becomes superficial toward the caudal direction. At its distal end, the sacral hiatus appears with the sacrococcygeal ligament as its roof and the anterior wall of the sacral canal as its floor. Between the roof and the floor is the continuation of the epidural space (fig 10B). Pivoting the transducer to the axial plane allows visualization of the sacral cornua, two hyperechoic bony prominences located besides the sacral hiatus (fig 10C). Relocating the transducer more laterally, a bony discontinuity emerges on 

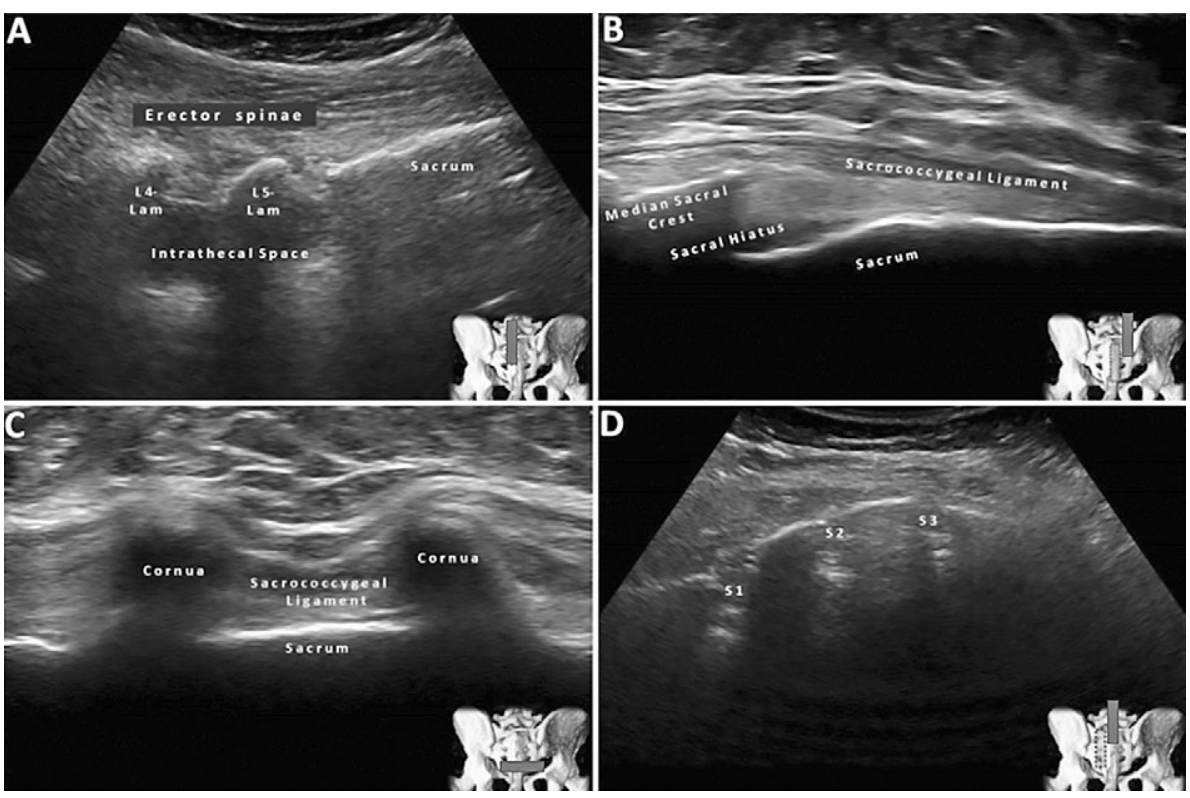

Fig 10. Sagittal ultrasound imaging at the L5/S1 interlaminar (A) and sacral hiatus (B) levels, axial imaging of the sacral hiatus (C) and sagittal imaging of the sacral foramina 1 (S1) to 3 (S3) (D). Lam, lamina

the posterior sacrum, which is the $\mathrm{S} 4$ sacral foramen. The S3, S2 and S1 sacral foramina can be recognized sequentially by backing the transducer cranially (fig 10D) [27].

\section{Conclusion}

As in scanning the extremities, the spine can also be approached by using US imaging in a systematic manner. Frequent interchange between the linear and curved transducers would be anticipated for optimal visualization of the spine and the relevant neuromuscular structures. Doppler imaging is also mandatory for identifying vital vasculature near the targeted region(s).

\section{Acknowledgment}

The present study is supported by the National Taiwan University Hospital, Bei-Hu Branch, Ministry of Science and Technology (MOST 106-2314-B-002-180MY3) and Taiwan Society of Ultrasound in Medicine.

\section{Conflict of interest: none}

\section{References}

1. Özçakar L, Carli AB, Tok F, Tekin L, Akkaya N, Kara M. The utility of musculoskeletal ultrasound in rehabilitation settings. Am J Phys Med Rehabil 2013;92:805-817.

2. Özçakar L, Kara M, Chang KV, et al. Nineteen reasons why physiatrists should do musculoskeletal ultrasound: EUROMUSCULUS/USPRM recommendations. Am J Phys Med Rehabil 2015;94:e45-e49.
3. Özçakar L, Kara M, Chang KV, et al. EURO-MUSCULUS/ USPRM basic scanning protocols for hip. Eur J Phys Rehabil Med 2015;51:635-640.

4. Özçakar L, Kara M, Chang KV, et al. EURO-MUSCULUS/ USPRM basic scanning protocols for ankle and foot. Eur J Phys Rehabil Med 2015;51:647-653.

5. Özçakar L, Kara M, Chang KV, et al. EURO-MUSCULUS/ USPRM basic scanning protocols for elbow. Eur J Phys Rehabil Med 2015;51:485-489.

6. Özçakar L, Kara M, Chang KV, et al. EURO-MUSCULUS/ USPRM basic scanning protocols for shoulder. Eur J Phys Rehabil Med 2015;51:491-496.

7. Özçakar L, Kara M, Chang KV, et al. EURO-MUSCULUS/ USPRM basic scanning protocols for knee. Eur J Phys Rehabil Med 2015;51:641-646.

8. Özçakar L, Kara M, Chang KV, et al. EURO-MUSCULUS/ USPRM basic scanning protocols for wrist and hand. Eur J Phys Rehabil Med 2015;51:479-484.

9. Kara M, Kaymak B, Ulasli AM, et al. Sonographic guide for botulinum toxin injections of the upper limb: EUROMUSCULUS/USPRM spasticity approach. Eur J Phys Rehabil Med 2018;54:469-485.

10. Kaymak B, Kara M, Tok F, et al. Sonographic guide for botulinum toxin injections of the lower limb: EURO-MUSCULUS/USPRM spasticity approach. Eur J Phys Rehabil Med 2018;54:486-498.

11. Hung CY, Hsiao MY, Özçakar L, et al. Sonographic tracking of the lower limb peripheral nerves: a pictorial essay and video demonstration. Am J Phys Med Rehabil 2016;95:698-708.

12. Wu CH, Chang KV, Özçakar L, et al. Sonographic tracking of the upper limb peripheral nerves: a pictorial essay and video demonstration. Am J Phys Med Rehabil 2015;94:740-747. 
13. Chang KV, Sahin Onat S, Lee CW, Kara M, Hung CY, Özçakar L. EURO-MUSCULUS/USPRM basic scanning protocols revisited in children. Eur J Phys Rehabil Med 2016;52:887-901.

14. Waxenbaum JA, Futterman B. Anatomy, back, vertebrae, thoracic. In: StatPearls. Treasure Island: StatPearls Publishing; (FL) 2017.

15. Waxenbaum JA, Futterman B. Anatomy, back, vertebrae, cervical. In: StatPearls. Treasure Island: StatPearls Publishing; (FL) 2017.

16. Waxenbaum JA, Futterman B. Anatomy, back, vertebrae, lumbar. In: StatPearls. Treasure Island: StatPearls Publishing; (FL) 2017.

17. Latchaw RE, Albers SL. Imaging the cervical vasculature. Prog Cardiovasc Dis 2017;59:555-584.

18. Colman MW, Hornicek FJ, Schwab JH. Spinal cord blood supply and its surgical implications. J Am Acad Orthop Surg 2015;23:581-591.

19. Chang KV, Lin CP, Hung CY, Özçakar L, Wang TG, Chen WS. Sonographic nerve tracking in the cervical region: a pictorial essay and video demonstration. Am J Phys Med Rehabil 2016;95:862-870.

20. Chang KV, Lin CP, Wu WT, Özçakar L. Ultrasound-guided selective cervical root injection for postherpetic neuralgia. Am J Phys Med Rehabil 2017;96:e189-e190.
21. Chang KV, Wu WT, Özçakar L. Ultrasound-guided interventions of the cervical spine and nerves. Phys Med Rehabil Clin N Am 2018;29: 93-103.

22. Chang KV, Wu WT, Özçakar L. Ultrasound-guided C7 cervical medial branch block using the in-plane approach. Am J Phys Med Rehabil 2017;96:e164.

23. Kaymak B, Kara M, Gurcay E, Özçakar L. Sonographic guide for botulinum toxin injections of the neck muscles in cervical dystonia. Phys Med Rehabil Clin N Am 2018;29:105-123.

24. Chang KV, Lin CP, Lin CS, Wu WT, Karmakar MK, Özçakar L. Sonographic tracking of trunk nerves: essential for ultrasound-guided pain management and research. J Pain Res 2017;10:79-88.

25. Chin KJ, Karmakar MK, Peng P. Ultrasonography of the adult thoracic and lumbar spine for central neuraxial blockade. Anesthesiology 2011;114:14591485 .

26. Gurcay E, Kara M, Kaymak B, Soylu AR, Özçakar L. Prompt sonographic scanning and quantification of lumbar muscles: seeing the butterfly. Scand J Med Sci Sports 2018;28:1314.

27. Kao SC, Lin CS. Caudal epidural block: an updated review of anatomy and techniques. Biomed Res Int 2017;2017:9217145. 